\title{
Composición botánica de la dieta del guanaco (Lama guanicoe) en la Reserva Corazón de la Isla, Tierra del Fuego (Argentina): utilización estacional de Nothofagus spp.
}

\author{
Diet composition of guanacos (Lama guanicoe) in Corazón de la Isla Reserve, \\ Tierra del Fuego (Argentina): seasonal utilization of Nothofagus spp.
}

\author{
Nadia Arias a,b,c*, Sandra Feijóo a ${ }^{\text {a }}$ Pamela Quinteros ${ }^{\text {c,d }}$, José Bavad \\ a Universidad Nacional de la Patagonia San Juan Bosco, Facultad de Ciencias Naturales, \\ Departamento de Biología, Comodoro Rivadavia, Argentina. \\ *Autor de correspondencia: ${ }^{\text {b }}$ Grupo de Estudios Biofísicos y Eco-fisiológicos (GEBEF), Departamento de Biología, \\ Facultad de Ciencias Naturales, Comodoro Rivadavia, Argentina, tel.: 0297-154300765, ns_arias@yahoo.com.ar \\ ${ }^{\mathrm{c}}$ Consejo Nacional de Investigaciones Científicas y Técnicas (CONICET), Argentina. \\ ${ }^{\mathrm{d}}$ Centro de Investigación y Extensión Forestal Andino Patagónico (CIEFAP), Esquel, Argentina.
}

\begin{abstract}
SUMMARY
Guanaco (Lama guanicoe) is one of the most characteristic species of Tierra del Fuego province. The damage produced by guanacos in the regeneration of lenga (Nothofagus pumilio) in ecotonal forests has been documented. The aim of this study were to determine the diet composition of guanaco in a forest area that includes a portion of wetlands and to estimated seasonal presence and frequency of Nothofagus spp. in the diet. The guanaco diet was assessed by fecal micro-histological analyses. The proportion of species consumed by guanaco showed significant differences throughout the year. Caespitose grasses and rhizomatous grasses represent $52 \%$ of the annual diet of guanaco. Festuca spp., Poa spp., Carex spp. and Uncinia lechleriana are, within their respective groups, the most consumed items. Consideration of non epidermal tissue as phellem is essential to identify Nothofagus spp. in the diet of guanaco in Tierra del Fuego. The presence of Nothofagus spp. is constant and abundant throughout the year and represents the $29 \%$ of the annual guanaco diet in Tierra del Fuego. Guanaco consumed saplings of Nothofagus spp. with presence of leaves and/or buds at different times of the year. The major consumption of Nothofagus spp. seedlings by guanaco could adversely affect the development of regeneration, and this aspect should be considered in the management and implementation of forestry or livestock activities in the forest.
\end{abstract}

Key words: fecal micro-histological analyses -tree saplings - non epidermal tissues

\section{RESUMEN}

El guanaco (Lama guanicoe) representa una de las especies más características de la fauna de Tierra del Fuego. En bosques ecotonales se ha documentado el daño producido por el guanaco en la regeneración de la Nothofagus pumilio (lenga). Los objetivos del trabajo fueron determinar la composición botánica de la dieta anual del guanaco en un área de bosque que incluye un sector de humedales y estimar presencia estacional de Nothofagus spp. en la dieta. La dieta se evaluó mediante el método microhistológico de heces. La proporción de especies consumidas por el guanaco presenta diferencias significativas entre los distintos meses del año. Las gramíneas y graminoideas representan el $52 \%$ de la dieta anual. Festuca spp., Poa spp., Uncinia lechleriana y Carex spp., son dentro de sus respectivos grupos los géneros más consumidos por el guanaco. La presencia de Nothofagus spp. es constante y abundante durante todo el año y representa el $29 \%$ de la dieta anual. La consideración de tejidos no epidérmicos es fundamental para identificar Nothofagus spp. en la dieta del guanaco en Tierra del Fuego. El guanaco consume ramas jóvenes de renovales de Nothofagus spp. con presencia de hojas o yemas en las diferentes épocas del año. El importante consumo de renovales de Nothofagus spp. podría afectar negativamente el desarrollo de la regeneración de estos bosques, por lo cual consideramos que este aspecto debe ser tenido en cuenta en la planificación y ejecución de actividades forestales y ganaderas en el bosque.

Palabras clave: análisis microhistológico de heces, renovales, tejidos no epidérmicos.

\section{INTRODUCCIÓN}

El guanaco (Lama guanicoe Müller) es el más grande de los camélidos silvestres sudamericanos, su distribución abarca desde los $8^{\circ} \mathrm{S}$ en Perú hasta casi los $55^{\circ} \mathrm{S}$ en la Isla de Tierra del Fuego (Puig et al. 1997) y representa una de las especies más características de la fauna de Tierra del Fuego. La densidad media de guanacos estimada fue de 0,97 individuos $\mathrm{km}^{-2}$ registrándose un total de 20.000 individuos en la Isla de Tierra del Fuego para el año 1995 (Montes et al. 2000). El último censo realizado para la zona central de la isla en el año 2008 muestra una den- 
sidad de 2,05 individuos $\mathrm{km}^{-2} \mathrm{y}$ un total de 14.000 individuos en dicha área (Schiavini et al. 2009). Se sabe que el guanaco realiza movimientos estacionales desde zonas altas (bosques) en verano hacia zonas más bajas (costeras) libres de nieve en invierno y otoño (Bonino y Fernández 1994). Este patrón de movimiento estacional podría estar modificado por el desplazamiento competitivo por parte de la oveja quienes ocupan las zonas bajas de estepa (Raedeke 1982, Baldi et al. 2001, 2004). Actualmente, la mayor abundancia relativa de guanacos se encuentra en el área de ecotono bosque estepa y de bosques, concentrando el $84 \%$ de la población estimada para la región (Montes et al. 2000). Las zonas de ecotono ofrecen un hábitat apropiado para el guanaco, en donde los manchones boscosos le brindan una adecuada cobertura de refugio y las zonas de claros asociadas a humedales le proporcionan el alimento necesario (Bonino y Sbriller 1992, Bonino y Fernández 1994). El guanaco es considerado por diferentes autores (Puig et al. 1997, Muñoz y Simonetti 2013, Barri et al. 2014) un herbívoro generalista que se alimenta de una amplia variedad de forraje. La dieta del guanaco varía estacionalmente en función de la productividad de su hábitat lo que genera que el uso y preferencia de especies leñosas se incremente cuando la disponibilidad de hierbas y pastos decrece (Puig et al. 1997). Al igual que la llama (Lama glama Muller 1776), el guanaco está adaptado a una baja calidad de forraje con abundantes fibras, presentando una gran eficiencia digestiva (San Martin y Bryant 1989). Según Pinto Jiménez et al. (2010), a pesar de que el guanaco no es considerado un rumiante, puede retener el alimento en su tracto digestivo por un largo período de tiempo, lo que lo hace altamente eficiente para digerir alimentos de baja calidad forrajera.

En las zonas de bosques ecotonales se ha documentado el daño producido por el guanaco en la regeneración de bosques de Nothofagus pumilio (Poep et. Endl) Krasser (lenga) (Martínez Pastur et al. 1999a, Caviers y Fajardo 2005) donde su dieta estaría compuesta casi en un $15 \%$ por ramas de árboles y arbustos (Raedeke 1982). En Tierra del Fuego se ha evidenciado que las especies del género Nothofagus son las más consumidas dentro de los árboles (Raedeke 1982, Bonino y Pelliza Sbriller 1991, Muñoz 2008, Soler et al. 2011, 2013). Los bosques de Nothofagus spp. (Nothofagaceae) ocupan 635.000 ha en el sector argentino de Tierra del Fuego y algunos son intensamente aprovechados por el interés en su madera. La regeneración de los bosques de Nothofagus spp. ha sido estudiada tanto desde una perspectiva ecológica (Rebertus y Veblen 1993) como silvícola (Martínez Pastur et al. 1999a). Sin embargo, las formas actuales de aprovechamiento plantean algunos interrogantes acerca de la capacidad de respuesta regenerativa, sobre todo si las áreas explotadas están expuestas al ramoneo de herbívoros salvajes o domésticos (Martínez Pastur et al. 1999a). El ramoneo por el guanaco puede constituir una seria amenaza para la regeneración del bosque aprovechado y, probablemente, de sectores de bosque virgen cercanos a las áreas preferidas de pastizales (Martínez Pastur et al. 1999b). Es por esto que es preciso analizar la incidencia del ramoneo sobre los bosques de Nothofagus spp. a una escala de tiempo más amplia para conocer su efecto en la dinámica y la producción forestal (Pulido et al. 2000).

La dieta de herbívoros silvestres y domésticos puede ser evaluada mediante diferentes técnicas, entre ellas el método microhistológico, el cual consiste en el análisis microscópico de los restos no digeridos de las plantas consumidas. Dicha técnica puede realizarse en contenidos estomacales o en heces, en donde la identificación se basa en el reconocimiento de la epidermis vegetal (principalmente foliar), tejido rico en caracteres constantes para cada taxón y protegido por la cutícula de los efectos de la digestión. Sin embargo, algunos tejidos no epidérmicos que resisten el proceso digestivo, como el esclerénquima y algunos tipos de parénquima pueden producir restos identificables útiles en el análisis de la dieta (Latour y Pelliza Sbriller 1981). Considerar los tejidos no epidérmicos en el análisis microhistológico de heces permite obtener resultados más representativos de la dieta de herbívoros (Sepúlveda Palma et al. 2004).

El objetivo general del presente estudio es determinar la composición botánica de la dieta del guanaco, durante diferentes épocas del año, en un sistema conformado por un bosque de Nothofagus spp., antiguamente utilizado forestalmente, y humedales con elevada oferta forrajera. Como objetivo específico se plantea estimar presencia y frecuencia estacional de Nothofagus spp. en la dieta del guanaco.

La hipótesis general del trabajo es que la dieta del guanaco está compuesta principalmente por especies pertenecientes a los grupos gramíneas y graminoideas y que el consumo de Nothofagus spp. es constante a lo largo del año.

\section{MÉTODOS}

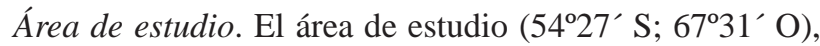
está comprendida dentro de la reserva Corazón de la Isla, provincia de Tierra del Fuego, Argentina (figura 1).

La reserva Corazón de la Isla abarca aproximadamente una superficie de 100.000 ha. Comprende una región de cordillera y una pequeña porción de ecotono o transición. La región cordillerana se caracteriza por bosques densos de $N$. pumilio y guindo (Nothofagus betuloides (Mirb) Oerst.) y la región de ecotono se caracteriza por manchones de bosque de $N$. pumilio y (Nothofagus antarctica (G. Forster) Oerst. (ñire) con sectores de humedales denominados tradicionalmente "vegas". Según el inventario y zonificación de la Reserva Corazón de la Isla (Gigli 2001) el porcentaje de especies clasificadas para la región corresponde a un $3 \%$ para forestales, un $39 \%$ para dicotiledóneas, un $21 \%$ para monocotiledóneas y un $37 \%$ a inferiores (dos pteridofitas, 22 musgos y una hepática). La zona de muestreo está ubicada en el límite Noreste de la Reserva 


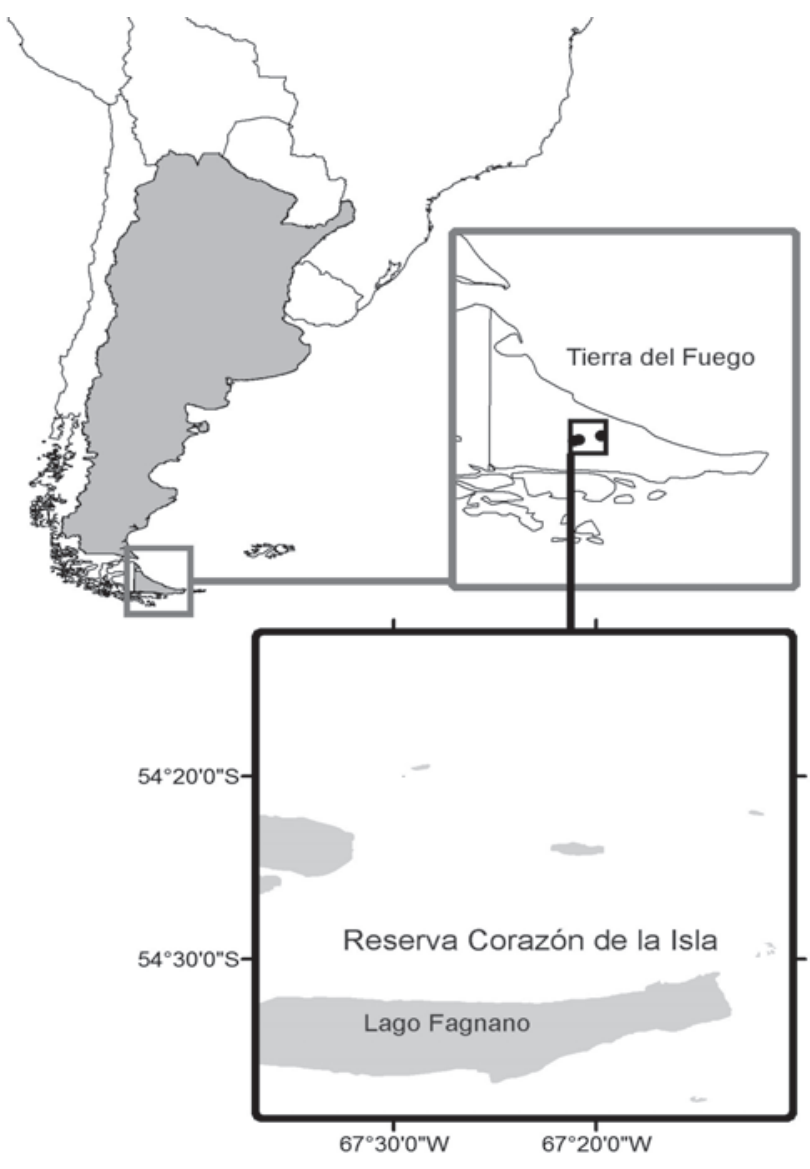

Figura 1. Reserva Corazón de la Isla, Tierra del Fuego, Argentina. Corazón de la Isla Reserve, Tierra del Fuego, Argentina.

y se caracteriza por la presencia de bosques deciduos de $N$. pumilio y $N$. antarctica, con predominio de la primera, ambas especies endémicas de los bosques templados de Sudamérica (Donoso 2006). Para una región similar en la zona central de Tierra del Fuego, Soler et al. (2013) determinaron que los bosques de Nothofagus spp. representaban un $71,3 \%$, de los cuales $19,4 \%$ correspondían a Nothofagus antarctica y $51,9 \%$ a Nothofagus pumilio. El clima en la Isla Grande de Tierra del Fuego se halla comprendido dentro de la clasificación climática "templado-fría”, con categoría de subhúmedo en el Norte y oceánica en los bosques cordilleranos. En la Reserva Corazón de la Isla, las temperaturas medias anuales oscilan entre los $4,5^{\circ} \mathrm{C}$ y las precipitaciones medias mensuales se encuentran alrededor de los $47 \mathrm{~mm}$. Los bosques de $N$. pumilio y $N$. antarctica del área de estudio han sido aprovechados forestalmente hace aproximadamente 50 años mediante floreos que consistían en la extracción selectiva de los mejores individuos maderables. En el área de estudio no se encuentra ganado ovino o vacuno, de manera que el guanaco constituye el único herbívoro de gran tamaño presente en la zona con poblaciones estables a lo largo del año.
Análisis microhistológico de heces. Se realizaron relevamientos de vegetación y se colectaron muestras de heces y de plantas. Esta actividad estuvo a cargo de personal de la Dirección de Áreas Protegidas y Biodiversidad de la provincia de Tierra del Fuego. Los ejemplares vegetales de referencia fueron depositados en el Herbario Regional Patagónico (HRP) de la Facultad de Ciencias Naturales, UNPSJB. Los muestreos se realizaron durante los meses de abril (otoño), junio (invierno), septiembre (primavera temprana) y noviembre (primavera tardía) del año 2009 y enero (verano) de 2010. En cada estación se colectaron heces frescas en cantidades equivalentes y representativas de 10 bosteaderos y de 10 bosteos individuales, todos ubicados dentro del bosque. Las heces frescas colectadas en cada estación constituyeron una única muestra compuesta utilizada para el análisis microshistológico. En el laboratorio se procesaron los materiales y se confeccionaron patrones de referencia de las plantas presentes en el área de estudio teniendo en cuenta el relevamiento de vegetación presente en cada estación. Se realizaron diafanizados y moliendas de hojas de $N$. pumilio y N. antarctica, así como también moliendas y cortes transversales de tallo de ambas especies. Las muestras compuestas de heces se secaron en estufa a $60^{\circ} \mathrm{C}$ y se molieron con un molino analítico (IKA A11), obteniendo un tamaño final de partícula de $1 \mathrm{~mm}$. La composición botánica de la dieta se determinó por medio del método microhistológico de heces (Latour y Pelliza Sbriller 1981) identificando fragmentos vegetales epidérmicos y no epidérmicos (Sepúlveda Palma et al. 2004). Se elaboraron cinco preparados y se analizaran 100 campos microscópicos por muestra. Se registraron las observaciones y los resultados se expresaron en porcentajes de frecuencia de los ítems determinados microhistológicamente, a nivel genérico y/o específico, agrupándose los mismos en seis grupos vegetales: árboles, gramíneas, graminoideas, hierbas, arbustos y líquenes. La cuantificación de los ítems integrantes de la dieta se efectuó según Holechek y Vavra (1981).

Composición botánica del sotobosque de Nothofagus spp. En el mes de noviembre (2009) se delimitó en la Reserva Corazón de la Isla una zona de bosque que incluía un humedal. A partir de allí se instalaron seis transectos perpendicular al borde y a 10, 20, 40, 80, 160 y $320 \mathrm{~m}$ de distancia. Al borde se ubicó una parcela rectangular de 50 x $10 \mathrm{~m}$ conteniendo cinco subparcelas de muestreo de $2 \mathrm{~m}^{2}$. En cada subparcela se determinaron las especies vegetales presentes y se estimó visualmente el porcentaje de cobertura.

Índices y análisis estadístico. La diversidad de especies vegetales en la dieta del guanaco se estimó utilizando el índice de Shannon Wiener. Para calcular la similitud de especies en la dieta entre las estaciones del año se aplicó el índice de Bray y Curtis. Los valores de frecuencia para cada grupo vegetal fueron analizados mediante el método de análisis de similitudes (ANOSIM) a fin de determinar 
las posibles diferencias entre los meses estudiados. Posteriormente los valores de frecuencia fueron usados para la construcción de una matriz de similitud, utilizando el método de escalamiento multidimensional (MDS) que permite el agrupamiento de las muestras. También se realizó un análisis de porcentajes de similitud (SIMPER) a fin de cuantificar la contribución de los grupos en la diferencia entre épocas del año. Las diferencias entre las dietas en los distintos meses del año se estimaron mediante un análisis de la varianza (ANDEVA) y las diferencias significativas se analizaron mediante la prueba de Tukey de comparaciones múltiples.

\section{RESULTADOS}

Dieta estacional del guanaco. En la Reserva Corazón de la Isla, la dieta anual del guanaco está compuesta por 17 especies vegetales. El índice de diversidad de Shannon-Wiener fue similar durante las diferentes épocas del año, presentando los valores más bajos en los meses de noviembre y enero (cuadro 1). Los grupos vegetales árboles, gramíneas y graminoideas fueron los más importantes en la dieta anual del guanaco. Las gramíneas y graminoideas representan el $52 \%$ de la dieta anual y los árboles representan el $29 \%$. Las principales gramíneas identificadas fueron Poa spp. y
Festuca spp.; dentro de las graminoideas Carex spp. y Uncinia lechleriana Steud. presentaron la mayor frecuencia. El género Nothofagus constituye la totalidad del ítem árboles y en el área de estudio las únicas especies registradas fueron $N$. pumilio y $N$. antarctica, con predominio de la primera. Ambas especies son anatómicamente muy similares y los caracteres que permiten distinguirlas a nivel foliar no fueron suficientes para ser utilizados como carácter diagnóstico en el análisis microhistológico de heces.

Similitud de especies y grupos de especies en la dieta del guanaco. La similitud de especies en la dieta entre estaciones fue alta, superando en todos los casos el $70 \%$ y con una correlación estadísticamente significativa $(P<0,01)$ entre las dietas de todos los meses. El valor de correlación más bajo $(0,31)$ se da entre los meses de junio y enero donde también se obtiene el menor valor de similitud $(0,74)$ (cuadro 2).

El análisis de porcentajes de similitud (SIMPER) mostró diferencias significativas entre las frecuencias de cada grupo vegetal a lo largo de año $(P<0,01)$. Dichas diferencias están dadas por los ítems árboles, gramíneas y graminoideas (cuadro 3). El método MDS (figura 2) representa el ordenamiento de las muestras en base a la similitud en la composición de la dieta. Las muestras de enero y abril, verano y

Cuadro 1. Frecuencia (\%) de especies vegetales presentes en heces de guanaco en diferentes meses del año en la Reserva Corazón de la Isla, Tierra del Fuego.

Relative frequency (\%) of plant species in feces of guanaco during different months of the year in Corazón de la Isla Reserve, Tierra del Fuego.

\begin{tabular}{lrrrrr}
\hline Especies & Abril & Junio & Septiembre & Noviembre & Enero \\
\hline Nothofagus spp. & 20,53 & 30,29 & 36,31 & 38,51 & 15,97 \\
Berberis spp. & 3,68 & 1,14 & 1,19 & 1,35 & 1,39 \\
Empetrum rubrum Vahl ex Willd. & 0,53 & 1,71 & - & - & - \\
Gaultheria mucronata (L.f.) Hook. et Arn. var. mucronata & 1,05 & 1,14 & 6,55 & - & - \\
Festuca magellanica Lam. & 10.53 & 6.29 & 5.95 & 6,08 & 2,78 \\
Phleum alpinum L. & 4,21 & 1,71 & 2,38 & 2,7 & 1,39 \\
Bromus spp. & - & - & - & - & 4,17 \\
Elymus spp. & 7,37 & 6,29 & 2,38 & - & 0,69 \\
Koeleria fueguina C.E. Calderón ex Nicora & 1,58 & 2,86 & 1,19 & - & - \\
Poa spp. & 12,63 & 19,43 & 16,67 & 1,35 & 20,83 \\
Carex spp. & 19,47 & 4 & 6,55 & 18,92 & 37,5 \\
Luzula spp. & 1,58 & - & - & - & 0,69 \\
Uncinia lechleriana Steud. & 7,37 & 6,86 & 5,36 & 12,84 & 0,69 \\
Acaena spp. & 7,89 & 13,14 & 1,79 & 7,43 & 2,08 \\
Viola magellanica G. Forst. & - & - & - & 8,11 & 11,11 \\
Blechnum penna-marina (Poir.) Kuhn & - & 1,71 & 4,17 & 1,35 & - \\
Líquenes & 1,58 & 3,43 & 9,52 & 1,35 & 0,69 \\
Índice de Shannon Wiener (H) & 2,25 & 2,13 & 2,06 & 1,84 & 1,80 \\
\hline
\end{tabular}


Cuadro 2. Índice de similitud de especies (Is) y coeficiente de correlación (Rs) en la dieta del guanaco entre los distintos meses del año meses del año en la Reserva Corazón de la Isla, Tierra del Fuego.

Species similarity index (Is) and correlation coefficient (Rs) in the diet of guanaco between different months of the year in Corazón de la Isla Reserve, Tierra del Fuego.

\begin{tabular}{lcc}
\hline \multicolumn{1}{c}{ Meses } & (Is) & (Rs) \\
\hline Abril-junio & 0,93 & 0,75 \\
Abril-septiembre & 0,89 & 0,65 \\
Abril-noviembre & 0,88 & 0,75 \\
Abril-enero & 0,81 & 0,72 \\
Junio-septiembre & 0,96 & 0,87 \\
Junio-noviembre & 0,80 & 0,67 \\
Junio-enero & 0,74 & 0,31 \\
Septiembre- noviembre & 0,92 & 0,74 \\
Septiembre -enero & 0,77 & 0,36 \\
Noviembre -enero & 0,83 & 0,44 \\
\hline
\end{tabular}

otoño respectivamente, aparecen formando un grupo. Otro grupo lo forman los meses de junio y septiembre, muestras que corresponden al invierno y primavera temprana; noviembre, primavera tardía, se separa presentando la mayor distancia con el mes de enero. Esta ordenación convalida las diferencias halladas para grupos vegetales en la dieta anual del guanaco. Según el análisis de la varianza (ANDEVA) también se encontraron interacciones altamente significativas entre los factores analizados (F 9,63, $P<0,001)$. El consumo de gramíneas se mantuvo constante durante todo el año, descendiendo a un valor cercano al $11 \%$ en el mes de noviembre, donde se observó un aumento de las graminoideas (32\%), representadas en su mayoría por Uncinia lechleriana Steud., especie presente en el sotobosque de $N$. pumilio. Mientras que el alto consumo de este ítem registrado en los meses de enero (39\%) y abril (20\%), está representado principalmente por Carex spp. y otras especies propias de las vegas. Durante los meses de junio, septiembre y noviembre el consumo de árboles fue importante, presentando valores mayores al $30 \%$, mientras que, en enero y abril descendió por debajo del $20 \%$. Paralelamente se observó un aumento en el porcentaje de gramíneas (37 \% y $30 \%$, respectivamente). La presencia de hierbas en la dieta aumentó en los meses de noviembre y enero, aunque representadas por especies diferentes. En noviembre se presentaron cantidades importantes de Acaena spp. y en enero de Viola magellanica (G. Forst.). Los arbustos se mantuvieron constante durante todos los meses del año, con un promedio anual del $5 \%$, y estuvieron representados en su mayoría por Berberis spp. Los líquenes estuvieron presentes en la dieta del guanaco con un promedio anual del $3 \%$.

Presencia de Nothofagus spp. en la dieta estacional del guanaco. La presencia de Nothofagus spp. fue constante y abundante durante todas las estaciones del año en la dieta del guanaco en la Reserva Corazón de la Isla y representó el $29 \%$ de la dieta anual. Los valores más altos de Notho-

Cuadro 3. Similitud de grupos vegetales en la dieta del guanaco entre meses del año en la Reserva Corazón de la Isla, Tierra del Fuego. Contribución de cada uno de los grupos vegetales a la diferenciación entre los distintos meses.

Similarity of plant groups in the guanaco diet among different months of the year in the Corazón de la Isla Reserve, Tierra del Fuego. Contribution of each plant groups to the differences found.

\begin{tabular}{lcccccc}
\hline \multirow{2}{*}{ Periodo } & \multicolumn{7}{c}{ Grupos vegetales (\%) } \\
\cline { 2 - 7 } & Árboles & Gramíneas & Graminoideas & Hierbas & Arbustos & Líquenes \\
\hline Abril - Junio & 16,42 & 15,18 & 41,90 & 13,15 & 7,14 & $\mathrm{X}$ \\
Abril - Sep. & 22,79 & 20,00 & 37,91 & 9,60 & $\mathrm{X}$ & $\mathrm{X}$ \\
Abril - Nov. & 16,05 & 47,57 & 12,24 & 14,94 & $\mathrm{X}$ & $\mathrm{X}$ \\
Abril - Enero & 24,25 & 29,42 & 21,73 & 12,09 & 9,85 & $\mathrm{X}$ \\
Junio - Sep. & 22,11 & 20,58 & 16,06 & 21.78 & 12,03 & $\mathrm{X}$ \\
Junio - Nov. & 11,92 & 41,08 & 26,65 & 9,85 & $\mathrm{X}$ & 5,72 \\
Junio - Enero & 29,14 & 17,06 & 35,27 & 9,38 & $\mathrm{X}$ & $\mathrm{X}$ \\
Sep. - Nov. & 14,23 & 29,11 & 27,70 & 18,46 & 7,59 & $\mathrm{X}$ \\
Sep. - Enero & 35,16 & $\mathrm{X}$ & 34,39 & 9,67 & 10,80 & $\mathrm{X}$ \\
Nov. - Enero & 33,99 & 27,23 & 17,46 & 13,58 & $\mathrm{X}$ & $\mathrm{X}$ \\
\hline
\end{tabular}

$\mathrm{X}$ indica los grupos que menos contribuyen a las diferencias encontradas $(<5 \%)$.

$\mathrm{X}$ indicates the groups that contribute the least to the differences found $(<5 \%)$. 


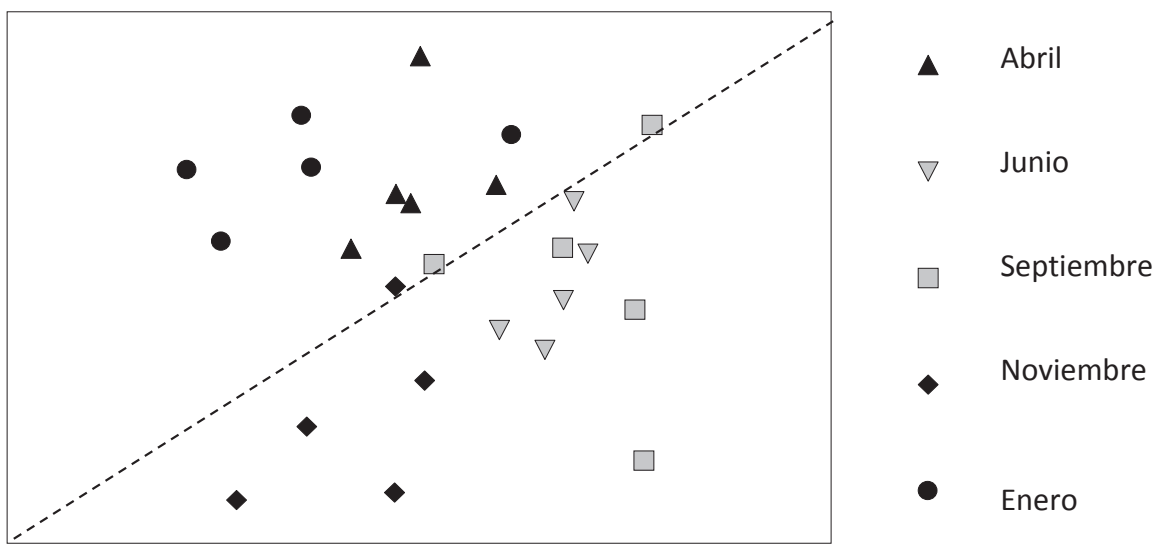

Figura 2. Ordenamiento de las muestras de heces, por el método "multidimensional scaling" (MDS). Un estrés de 0,14 significa que la representación bidimensional es útil.

Ordering of feces samples for the "multidimensional scaling" (MDS) method. A stress of 0.14 means the two-dimensional representation is useful.

fagus spp. se registraron durante los meses de invierno y primavera. Durante los meses de primavera y verano se identificaron mayormente fragmentos pertenecientes a tejidos epidérmicos con células rectangulares de paredes delgadas, estomas anomocíticos, tricomas unicelulares simples y drusas. En algunos fragmentos se observaron restos de haces vasculares con vaina parenquimática. Durante los meses de invierno, período en el cual la frecuencia de Nothofagus spp. fue alta, se identificaron mayormente tejidos no epidérmicos conformados por células pertenecientes al súber (peridermis), que se reconocen por su ordenamiento característico y por encontrarse asociadas a epidermis con tricomas unicelulares. Dichos fragmentos pertenecen a ramas jóvenes de renovales de Nothofagus spp., que son las que constituyen la oferta forrajera en el período de reposo.

Diversidad de especies vegetales en el sotobosque de Nothofagus spp. Se registraron 29 especies en el sotobosque de Nothofagus spp. de las cuales una es arbórea (renovales de $N$. pumilio), dos arbustivas, siete herbáceas exóticas y las restantes herbáceas nativas (cuadro 4). La cobertura total del sotobosque fue en promedio de $64,2 \%$.

\section{DISCUSIÓN Y CONCLUSIONES}

Dieta estacional del guanaco. La proporción de especies consumidas por el guanaco presenta diferencias significativas entre los distintos meses del año comportándose en la zona de estudio como un herbívoro generalista. Las gramíneas, graminoideas y árboles representan los grupos funcionales más importantes en la dieta anual del guanaco siendo Festuca spp., Poa spp., Uncinia lechleriana, Carex spp. y Nothofagus spp. los géneros más consumidos por el guanaco. El consumo de graminoideas y gramíneas posiblemente disminuya en invierno y primavera temprana debido a la presencia de nevadas intensas las cuales impiden el acceso de los animales a las zonas de humedales o vegas. En verano y otoño cuando las condiciones ambientales son más favorables, el guanaco hace uso de las vegas aumentando la proporción de ítems pertenecientes a estos sectores. En primavera tardía y verano aumenta el consumo de graminoideas y hierbas, representadas mayormente por especie abundantes en el sotobosque. La presencia de Nothofagus spp. es constante y abundante durante todas las estaciones del año en la dieta del guanaco en la Reserva Corazón de la Isla.

Diversos estudios de dieta estacional de guanaco realizados en Tierra del Fuego para áreas de ecotono bosqueestepa registran valores similares para gramíneas y graminoideas a los obtenidos en el presente trabajo. La diversidad de especies en la dieta fue similar durante todos los meses del año, consistente con lo obtenido por Bonino y Pelliza Sbriller (1991), como así también la frecuencia de líquenes en heces, el grupo de menor importancia en la dieta del guanaco. Se coincide también con estos autores en que las cantidades obtenidas pueden haberse subestimado debido a la alta digestibilidad de los mismos. Raedeke (1980) en el sector chileno de Tierra del Fuego y trabajando con contenido estomacal registró un valor anual del $7 \%$. La proporción anual de árboles obtenida en este estudio es mayor a la registrada por Bonino y Pelliza Sbriller (1991) y Soler et al. (2011, 2013) para una zona similar y por Muñoz (2008) y Muñoz y Simonetti (2013) para una zona boscosa en la región chilena de Tierra del Fuego. Esta diferencia se sustentaría en la identificación de tejidos no epidérmicos en los análisis microhistológicos de heces.

Presencia de Nothofagus spp. en la dieta estacional del guanaco. Durante el invierno y la primavera se registran las mayores frecuencias de árboles en la dieta del guanaco representados por $N$. pumilio y $N$. antarctica, ambas especies deciduas. Esto sugeriría que el guanaco consume 
Cuadro 4. Listado de especies registradas en el sotobosque de N. pumilio ordenadas por forma de vida (H: herbácea, A: arbusto, Ar: arbórea).

List of species registered in the underwood of $N$. pumilio ordered by lifestyle. (H: herbaceous, A: bush, Ar: tree).

\begin{tabular}{|c|c|c|}
\hline Familia & Especie & Forma de vida \\
\hline Apiaceae & Osmorhiza chilensis Hook. et Arn. & $\mathrm{H}$ \\
\hline \multirow[t]{4}{*}{ Asteraceae } & Adenocaulon chilense Less. & $\mathrm{H}$ \\
\hline & Senecio tricuspidatus Hook. et Arn. var. dumosus (Phil.) Cabrera & $\mathrm{H}$ \\
\hline & *Taraxacum officinale G. Weber ex F.H. Wigg. & $\mathrm{H}$ \\
\hline & Leptinella scariosa Cass. & $\mathrm{H}$ \\
\hline Berberidaceae & Berberis microphylla G. Forst. & A \\
\hline Blechnaceae & Blechnum penna-marina (Poir.) Kuhn & $\mathrm{H}$ \\
\hline Brassicaceae & Cardamine gracialis( G. Forst.) DC. & $\mathrm{H}$ \\
\hline Caryophyllaceae & *Cerastium fontanum Baumg. ssp. vulgare (Hartmann) Greuter et Burdet & $\mathrm{H}$ \\
\hline Cyperaceae & Uncinia lechleriana Steud. & $\mathrm{H}$ \\
\hline Euphorbiaceae & Dysopsis glechomoides (A. Rich.) Müll. Arg. & $\mathrm{H}$ \\
\hline Grossulariaceae & Ribes magellanicum Poir.ssp. magellanicum & A \\
\hline Juncaceae & Luzula alopecurus Desv. & $\mathrm{H}$ \\
\hline Nothofagaceae & Nothofagus pumilio (Poepp. et Endl.) Krasser & $\mathrm{Ar}$ \\
\hline Plantaginaceae & *Veronica serpyllifolia L. & $\mathrm{H}$ \\
\hline \multirow[t]{7}{*}{ Poaceae } & Bromus catharticus Vahl. & $\mathrm{H}$ \\
\hline & *Dactylis glomerata L. & $\mathrm{H}$ \\
\hline & Elymus angulatus J. Presl & $\mathrm{H}$ \\
\hline & Festuca magellanica Lam. & $\mathrm{H}$ \\
\hline & Koeleria fueguina C.E. Calderón ex Nicora & $\mathrm{H}$ \\
\hline & Phleum alpinum L. & $\mathrm{H}$ \\
\hline & *Poa pratensis L ssp. pratensis & $\mathrm{H}$ \\
\hline Polygonaceae & *Rumex acetosella L. & $\mathrm{H}$ \\
\hline \multirow[t]{4}{*}{ Rosaceae } & Acaena magellanica (Lam.) Vahl & $\mathrm{H}$ \\
\hline & Acaena ovalifolia Ruiz et Pav. & $\mathrm{H}$ \\
\hline & Geum magellanicum Comm. ex Pers. & $\mathrm{H}$ \\
\hline & Rubus geoides Sm. & $\mathrm{H}$ \\
\hline Rubiaceae & *Galium aparine L. & $\mathrm{H}$ \\
\hline Violaceae & Viola magellanica G. Forst. & $\mathrm{H}$ \\
\hline
\end{tabular}

* especies exóticas.

* exotic species.

no solo hojas sino ramas jóvenes de renovales de Nothofagus spp. con presencia de yemas vegetativas. Trabajos realizados en la misma área de estudio han registrado un importante daño por ramoneo de guanaco en los renovales de $N$. pumilio (Quinteros 2014). Dicha autora evidenció que aproximadamente la mitad del total de los renovales evaluados presentaba el ápice y/o la mayoría de las ramas ramoneadas, y que el $90 \%$ de los renovales entre $10 \mathrm{~cm}$ y $2 \mathrm{~m}$ de altura presentaba daño intenso por ramoneo. Evi- dencias similares también han sido detectados por Pulido et al. (2000) y Cavieres y Fajardo (2005) en bosques de $N$. pumilio presentes en Tierra del Fuego.

Los resultados del presente trabajo difieren de los obtenidos por Arroyo et al. (1996), Muñoz (2008) y Muñoz y Simonetti (2013), quienes afirman que el guanaco se alimenta sólo de hojas de lenga dejando intactas las ramas. San Martín y Bryant (1989) mencionan que la alta eficiencia del sistema digestivo del guanaco le permitiría hacer 
uso de especies leñosas para satisfacer sus requerimientos nutricionales, por lo cual se sugiere que el consumo de ramas con presencia de yemas le aportaría alimento durante el período invernal cuando la oferta de forraje en los humedales no está disponible por encontrarse congelada o cubierta de nieve. En este sentido se considera fundamental tener en cuenta en los análisis microhistológicos de heces los tejidos no epidérmicos con el fin de no subestimar el consumo de especies caducifolias como $N$. pumilio y $N$. antarctica.

Implicancias del ramoneo del guanaco en bosques antropizados de Nothofagus spp. El guanaco es un componente natural de los ecosistemas forestales de Tierra del Fuego y ha coexistidos con los bosques de Nothofagus spp. durante centurias, sin embargo, múltiples actividades antrópicas como el aprovechamiento forestal de los bosques, la introducción de ganado doméstico o la construcción de caminos (Schiavini et al. 2009) ocasionaron un mayor acceso de los animales a zonas de ecotono bosque-estepa. El intenso ramoneo de renovales jóvenes de Nothofagus spp. por parte del guanaco podría dificultar el proceso natural de regeneración de los bosques. En bosques aprovechados forestalmente, luego de la corta, se establece una abundante regeneración, sin embargo, el ramoneo persistente no permite el desarrollo normal de las plantas (Pulido et al. 2000). Los resultados del presente estudio evidencian un elevado consumo de ramas jóvenes de Nothofagus spp. durante la época invernal lo cual podría impedir el normal crecimiento de estos renovales. Los guanacos harían uso de recursos forrajeros en el bosque debido a un desplazamiento competitivo principalmente por el ganado introducido. Muñoz y Simonetti (2013) afirman que sin la presencia del ganado domestico el consumo de Nothofagus spp. en la dieta del guanaco disminuye en comparación con zonas donde coexiste con ganado ovino. Sin embargo, el bosque podría ser igualmente utilizado como refugio y dormidero, o como un corredor para las poblaciones migratorias (Raedeke 1980), lo cual sería más intenso en las zonas antropizadas del bosque.

El guanaco es un herbívoro que naturalmente incorpora importantes proporciones de especies leñosas en su dieta. En el área de estudio, la oferta de especies leñosas estuvo representada principalmente por $N$. pumilio. El consumo de renovales de esta especie podría afectar negativamente el desarrollo de la regeneración de estos bosques, por lo cual se considera que este aspecto debe ser tenido en cuenta en la planificación y ejecución de actividades forestales y ganaderas en el bosque.

\section{AGRADECIMIENTOS}

Los responsables del presente trabajo desean agradecer al personal de la Dirección de Áreas Protegidas y Biodiversidad de la Provincia de Tierra del Fuego, Argentina, y a los técnicos del CIEFAP y del Departamento de Biología General (UNPSJB) por la colaboración y la ayuda técnica brindada. Este trabajo pertenece a la Tesis de Licenciatura de la Licenciada Nadia Arias y fue posible gracias al financiamiento del PICTO 8/36767 obtenido por el Dr. José Bava y financiado por FONCYT Argentina.

\section{REFERENCIAS}

Arroyo M T, C Donoso, R Murúa, E Pisano, R Schlatter, I Serey. 1996. Toward an Ecologically Sustainable Forestry Project: Concepts, Analysis and Recommendations. Santiago, Chile. Departamento de Investigación y Desarrollo, Universidad de Chile. $253 \mathrm{p}$.

Baldi R, SD Albon, DA Elston. 2001. Guanacos and sheep: evidence for continuing competition in arid Patagonia. Oecologia 129: 561-570.

Baldi R, A Pelliza-Sbriller, D Lestón, S Albon. 2004. High potential for competition between guanacos and sheep in Patagonia. Journal of Wildlife Management 68: 924-938.

Barri FR, V Falczuk, AM Cingolani, S Díaz. 2014. Dieta de la población de guanacos (Lama guanicoe) reintroducida en el Parque Nacional Quebrada del Condorito, Argentina. Ecología Austral 24: 203-211.

Bonino N, A Pelliza Sbriller. 1991. Composición botánica de la dieta del guanaco (Lama guanicoe) en dos ambientes contrastantes de Tierra del Fuego, Argentina. Ecología Austral 1: 97-102.

Bonino N, A Pelliza Sbriller. 1992. Comparación de las dietas del guanaco, ovino y bovino en Tierra del Fuego, Argentina. Turrialba 41(4) 452:457.

Bonino N, E Fernández. 1994. Distribución general y abundancia de guanacos (Lama guanicoe) en diferentes ambientes de Tierra del Fuego, Argentina. Ecología Austral 4: 79-85.

Cavieres LA, A Fajardo. 2005. Browsing by guanaco (Lama guanicoe) on Nothofagus pumilio forest gaps in Tierra del Fuego, Chile. Forest Ecology and Management 204: 237248.

Donoso C. 2006. Las especies arbóreas de los bosques templados de Chile y Argentina. Autoecología. Valdivia, Chile. Ediciones Marisa Cuneo. 678 p.

Gigli SM. 2001. Inventario y zonificación de la Reserva Corazón de la Isla. Informe final Gobierno de Tierra del Fuego e Islas del Atlántico Sur. Ushuaia, Argentina. Subsecretaría de Planeamiento. 213 p.

Holechek J, M Vavra. 1981. The effects of slide and frequency observation numbers frequency on the precision of microhistological analysis. Journal of Range Management 34: 337-338.

Latour MC, A Pelliza Sbriller. 1981. Clave para la determinación de la dieta de herbívoros en el NO de Patagonia. Revista de Investigaciones Agropecuarias 16: 109-157.

Martínez-Pastur G, PL Peri, MC Fernández, G Staffieri, D Rodríguez. 1999a. Desarrollo de la regeneración a lo largo del ciclo del manejo forestal de un bosque de Nothofagus pumilo: 2. Incidencia del ramoneo de Lama guanicoe. Bosque 20(1): 47-53.

Martínez-Pastur G, PL Peri, MC Fernández, G Staffieri, D Rodríguez. 1999b. Desarrollo de la regeneración a lo largo del ciclo del manejo forestal de un bosque de Nothofagus pumilo: 1. Incidencia de la cobertura y el aprovechamiento o cosecha. Bosque 20(1): 39-46. 
Montes C, D Del Lamo, J Zanetti. 2000. Distribución de abundancia de guanaco (Lama guanicoe) en los distintos ambientes de Tierra del Fuego, Argentina. Mastozoología Neotropical. Journal of Neotropical Mammal 7(1): 23-31.

Muñoz A E. 2008. Guanaco (Lama guanicoe) browsing on lenga (Nothofagus pumilio) regeneration in Tierra del Fuego. Tesis de Magíster. Santiago, Chile. Facultad de Ciencias. Universidad de Chile. 31 p.

Muñoz AE, JA Simonetti. 2013. Diet of guanaco in sheep-free rangeland in Tierra del Fuego, Chile. Ciencia de Investigación Agraria 40(1): 185-191.

Pinto Jiménez C, C Martín Espada, M Cid Vázquez. 2010. Camélidos Sudamericanos: clasificación, origen y características. Revista Complutense de Ciencias Veterinarias 4 (1): 23-36.

Puig S, F Videla, M Cona. 1997. Diet and abundance of the guanaco (Lama guanicoe) in four habitats of northern Patagonia, Argentina. Journal of Arid Environments 36: 343-357

Pulido F, B Díaz, G Martinez Pastur. 2000. Incidencia del ramoneo del guanaco (Lama guanicoe Müeler) sobre la regeneración temprana en bosques de lenga (Nothofagus pumilio (Poepp et Endl) Krasser) de Tierra del Fuego, Argentina. Investigación Agraria de Sistemas y Recursos Forestales 9: 381-394.

Quinteros C P. 2014.Grandes herbívoros en bosques de lenga (N.pumilio): uso espacio-temporal de los recursos y sus efectos sobre la regeneración y el sotobosque. Tesis doctoral. Bariloche, Argentina. Facultad de Ciencias Naturales,
Universidad Nacional del Comahue. 172 p.

Raedeke KJ. 1980. Food habits of the guanaco (Lama guanicoe) of Tierra del Fuego, Chile. Turrialba 30: 177-181.

Raedeke KJ. 1982. Habitat use by guanacos (Lama guanicoe) and sheep on common range, Tierra del Fuego, Chile. Turrialba 32: 309-314.

Rebertus AJ, Veblen TT. 1993. Structure and tree-fall gap dynamics of old-growth Nothofagus forests in Tierra-del-Fuego, Argentina. Journal of Vegetation Science 4: 641-654.

San Martín F, FC Bryant. 1989. Nutrition of domesticated South American llamas and alpacas. Small Ruminant Research 2: 191-216.

Schiavini A, J Escobar, G Deferrari. 2009. Guanaco distribution and abundance in central Tierra del Fuego, Argentina. In X Congreso Internacional de Mastozoología, Mendoza, Argentina p. 144.

Sepúlveda Palma L, A Pelliza, M Manacorda. 2004. La importancia de los tejidos no epidérmicos en el microanálisis de la dieta de herbívoros. Ecología Austral 14: 31-38.

Soler Esteban R, G Martínez Pasteur, MV Lencinas, L Borelli. 2011. Differencial forage use between large native and domestic herbivores in Southern Patagonian Nothofagus forests. Agroforestry Systems 85: 397-409.

Soler Esteban R, G Martínez Pasteur, MV Lencinas, L Borelli. 2013. Sesonal diet of Lama guanicoe (Camelidae: Artiodactyla) in a heterogeneous landscape of South Patagonia. Bosque 34 (2): 129-141. 
